\title{
Erosión y pérdida de nutrientes en diferentes sistemas agrícolas de una microcuenca en la zona periurbana de la ciudad de Puebla, México
}

\author{
Erosion and loss of nutrients in different farming systems of a watershed \\ in the periurban area of the city of Puebla, Mexico
}

\author{
Rosalía Castelán Vega ${ }^{1,2}$, Leticia Citlaly López Teloxa ${ }^{2}$, J. Víctor Tamariz Flores ${ }^{1,2}$, \\ Gladys Linares Fleites ${ }^{1,2}$ y Abel Cruz Montalvo ${ }^{1}$
}

\footnotetext{
${ }^{1}$ Departamento de Investigación en Ciencias Agrícolas. Benemérita Universidad Autónoma de Puebla (BUAP). 14 Sur 6301, Col. San Manuel. 72590 Puebla, Puebla, México.

* Autor responsable (rosalia.castelan@correo.buap.mx)

${ }^{2}$ Posgrado en Ciencias Ambientales, Instituto de Ciencias-BUAP. Edificio IC 10. Cuidad Universitaria Colonia San Manuel. 72570 Puebla, Puebla, México.
}

\section{RESUMEN}

El suelo es un componente natural de gran importancia en la sostenibilidad de los ecosistemas, su degradación se debe, en la mayoría de los casos, al mal manejo bajo condiciones de ladera, lo que disminuye su productividad y su capacidad para sostener la vida humana. Se evaluó la producción de sedimentos y la pérdida de nutrientes durante el año 2013 en sistemas agrícolas representativos de la zona periurbana de la ciudad de Puebla, México; con el objetivo de ponderar el coeficiente de enriquecimiento de dichos sedimentos, y así identificar el sistema agrícola que menor perdida de fertilidad genera. Se instalaron parcelas delimitadas con dimensiones de $3 \mathrm{~m}$ de ancho y $9 \mathrm{~m}$ de largo con pendiente homogénea de $9 \%$ de inclinación, en los sistemas: maíz, maíz-calabaza y maíz-avena. Se recolectaron los sedimentos, a los cuales se les determinó la concentración de materia orgánica, nitrógeno y fósforo, según lo marca la NOM-021-SEMARNAT-2000. La producción de sedimentos y escorrentía fue significativamente mayor en el sistema agrícola de maíz $(54.6 \mathrm{~mm}$ y $37.9 \mathrm{Mg} \mathrm{ha}{ }^{-1}$, respectivamente) y menor en maízcalabaza. Los coeficientes de enriquecimiento de los sedimentos erosionados fueron en su mayoría, superiores a la unidad, destacando la materia orgánica con los coeficientes más altos. Se observó una fuerte correlación entre la producción de sedimentos y las pérdidas de materia orgánica, nitrógeno y fósforo, en los sistemas agrícolas. Los resultados obtenidos

Cita recomendada:

Castelán Vega, R., L. C. López Teloxa, J. V. Tamariz Flores, G. Linares Fleites y A. Cruz Montalvo. 2017. Erosión y pérdida de nutrientes en diferentes sistemas agrícolas de una microcuenca en la zona periurbana de la ciudad de Puebla, México. Terra Latinoamericana 35: 229-235. demuestran que el actual manejo de los cultivos agrícolas en la zona de estudio, favorecen la degradación de los suelos. De igual forma, los resultados generados son una base importante para establecer estrategias agrícolas y disminuir áreas degradadas en los espacios rurales de la zona periurbana de la ciudad de Puebla, México.

Palabras clave: uso de suelo, escorrentía, enriquecimiento de sedimentos.

\section{SUMMARY}

Soil is a natural component of great importance to ecosystem sustainability. Degradation of hillside soil conditions is due, in most cases, to poor management, which diminishes soil productivity and its ability to sustain human life. Production of sediment and nutrient loss during 2013 was evaluated in agricultural systems representative of the periurban zone of the city of Puebla, Mexico, to weight the enrichment coefficient of these sediments, and thus identify the agricultural system that generates less fertility loss. Delimited plots $3 \mathrm{~m}$ wide and $9 \mathrm{~m}$ long with a homogeneous slope of $9 \%$ were installed in maize, maize-squash and maizeoats crop systems. The sediments were collected, and concentrations of organic matter, nitrogen and phosphorus were determined, as marked by NOM-021SEMARNAT-2000. Sediment yield and runoff were significantly higher in maize $(54.6 \mathrm{~mm}$ and $37.9 \mathrm{Mg}$ $\mathrm{ha}^{-1}$, respectively) and lower in maize-squash plots.

Recibido: mayo de 2016. Aceptado: mayo de 2017. Publicado en Terra Latinoamericana 35: 229-235. 
The enrichment coefficients of the eroded sediments were mostly higher than one, and organic matter had the highest coefficients. There was a strong correlation between sediment production and losses of organic matter, nitrogen and phosphorus in agricultural systems. The results obtained demonstrate that the current management of agricultural crops in the study area favors soil degradation. Likewise, the results generated are an important basis for establishing agricultural strategies and reducing degraded areas of the rural periurban area of the city of Puebla, Mexico.

Index words: land use, runoff, nutrient.

\section{INTRODUCCIÓN}

La erosión del suelo es uno de los principales problemas ambientales a nivel mundial, sus efectos negativos se perciben no sólo en los ecosistemas que se desarrolla, ya que la pérdida progresiva de suelo y nutrientes constituyen un foco de contaminación difusa para otros sistemas, el ambiente y potencial biológico del suelo (FAO, 1997; Den Biggelaar et al., 2004; Almorox et al., 2010), por otro lado la erosión genera pérdidas económicas considerables a nivel nacional y, sobre todo, a los actores a nivel local (Cotler et al., 2011). Las valoraciones de erosión consideran, en su mayoría, sólo el suelo removido y desestiman las propiedades de los sedimentos erosionados (Leal et al., 2007), pese al conocimiento del enriquecimiento de los sedimentos con nutrientes del suelo (Sharpley, 1985). Tal es el caso de la microcuenca de San Andrés Azumiatla localizada en el municipio de Puebla, México; cuenta con alta actividad agrícola; sin embargo, el crecimiento constante y desmesurado de la población ha desplazado a los campesinos a las laderas de los lomeríos que rodean la ciudad, lo que ha originado un serio proceso erosivo en la región, pese a ello, nulos son los estudios acerca de esta degradación, que disminuye de forma paulatina y silenciosa la productividad y favorece la migración, por tanto el objetivo del presente trabajo fue evaluar la producción de sedimentos y la pérdida de nutrientes durante un año (2013) en tres sistemas agrícolas representativos de esta microcuenca.

\section{MATERIALES Y MÉTODOS}

La microcuenca de San Andrés Azumiatla se localiza al sur de la Presa Miguel Ávila Camacho, a $13 \mathrm{~km}$ de la ciudad de Puebla, México; enmarcada dentro de las coordenadas geográficas $18^{\circ} 48^{\prime} \mathrm{y}$ $18^{\circ} 56^{\prime} \mathrm{N}$ y $98^{\circ} 11^{\prime}$ y $98^{\circ} 17^{\prime} \mathrm{O}$; con un área aproximada de 5158 ha, a una altitud de $800 \mathrm{~m}$, precipitación media anual de $800 \mathrm{~mm}$ y temperatura promedio de $16{ }^{\circ} \mathrm{C}$ (INEGI, 2000). Se seleccionaron tres sistemas agrícolas representativos de la microcuenca: maíz, maíz-calabaza y maíz-avena; y se instalaron tres parcelas experimentales de $3 \mathrm{~m}$ de ancho por $9 \mathrm{~m}$ de largo por cada sistema, con inclinación de pendiente homogénea (9\%) (Almorox et al., 2010), durante el año 2013; para conocer las características de los suelos de las parcelas se tomaron por triplicado de cada parcela muestras compuestas a $30 \mathrm{~cm}$ de profundidad, según el muestreo conocido como "cinco de oro", el cual consiste en tomar una muestra en cada esquina y centro de la parcela, se mezclan juntas y se obtiene una muestra compuesta; posteriormente se etiquetan y trasladan al laboratorio para su posterior análisis.

Las parcelas experimentales se delimitaron con láminas galvanizadas; cada parcela tenía un sistema de drenaje paralelo a la pendiente, conectado a dos depósitos cerrados con capacidad de $65 \mathrm{~L}$ ubicados al pie de las parcelas, con la finalidad de obtener los sedimentos y escorrentía generados en los eventos pluviales (Adema et al., 2001). Posterior a cada lluvia se tomaron por triplicado muestras de sedimentos de $0.75 \mathrm{~L}$ cada una, se etiquetaron de acuerdoal uso de suelo, fecha y volumen total de escorrentía. En el laboratorio los sedimentos y las muestras compuestas de suelo se secaron, disgregaron y pasaron por tamiz de $2 \mathrm{~mm}$ de diámetro para realizar determinaciones de textura, materia orgánica, nitrógeno total y fósforo disponible de acuerdo a la NOM-021-SEMARNAT-2000 (NOM, 2000). La información analítica permitió calcular el coeficiente de enriquecimiento, definido como el cociente de la concentración de un producto químico " $X$ " en el sedimento transportado y la concentración de ese mismo producto químico " $X$ " en el suelo. Los registros de precipitación fueron a través de un pluviómetro ubicado a $200 \mathrm{~m}$ de cada parcela (Almoza et al., 2008). La producción de sedimentos por sistema 
agrícola se expresa en $\mathrm{Mg} \mathrm{ha}{ }^{-1}$. Se realizaron análisis de varianza y comparación de medias con la prueba de tukey con nivel de significancia del $5 \%$, correlaciones y regresión lineal entre las variables evaluadas.

\section{RESULTADOS Y DISCUSIÓN}

La precipitación acumulada durante el período de observación (un año) fue de $745 \mathrm{~mm}$, con un índice de erosividad de $10128.51 \mathrm{Mj} \mathrm{mm} \mathrm{ha}{ }^{-1}$, considerado como muy alto (Rivera y Gómez, 1991; Lobo y Bonilla, 2015); se registraron un total de 58 precipitaciones, de las cuales el 55\% superó los $10 \mathrm{~mm}$ de lluvia; el $10 \%$ fue mayor a los $30 \mathrm{~mm}$ y, sólo el 5\% rebasó los $50 \mathrm{~mm}$; las precipitaciones fueron variables y típicas del clima templado subhúmedo de la zona de estudio, con tormentas torrenciales breves, que causaron bajas tasas de infiltración, y alto riesgo de erosión. El Cuadro 1 presenta los promedios del volumen de escorrentía y producción de sedimentos durante el total de estas precipitaciones (58), hubo diferencias entre los sistemas agrícolas evaluados $(P \leq 0.05)$; la escorrentía $(54.6 \mathrm{~mm})$ y arrastre de sedimentos (37.9 $\mathrm{Mg} \mathrm{ha}{ }^{-1}$ ) fueron superiores en maíz y menores en maíz-calabaza, con $35.9 \mathrm{~mm}$ de escorrentía y 13.9 $\mathrm{Mg} \mathrm{ha}^{-1}$ de sedimentos, esto demostró que la siembra de maíz sin cobertura asociada favorece el proceso de erosión, ya que al sembrarse con avena o calabaza los efectos fueron menos severos. Los análisis de correlación demostraron que la acumulación de sedimentos estuvo asociada en los tres casos (maíz, maíz-calabaza y maíz-avena) a la precipitación, ya que la mayor cantidad de transporte de sedimentos
Cuadro 1. Sedimentos y escorrentía en los diferentes sistemas agrícolas evaluados.

\begin{tabular}{lcc}
\hline Agroecosistema & $\begin{array}{c}\text { Volumen de } \\
\text { escorrentía }\end{array}$ & $\begin{array}{c}\text { Producción de } \\
\text { Sedimento }\end{array}$ \\
\hline mm & $\mathrm{Mg} \mathrm{ha}^{-1}$ \\
Maíz & $54.6 \pm 1.26 \mathrm{a}$ & $37.9 \pm 0.46 \mathrm{a}$ \\
Maíz-Calabaza & $35.9 \pm 1.89 \mathrm{~b}$ & $13.9 \pm 0.76 \mathrm{~b}$ \\
Maíz-Avena & $42.8 \pm 1.46 \mathrm{c}$ & $17.7 \pm 0.94 \mathrm{c}$ \\
\hline
\end{tabular}

Letras distintas indican diferencias significativas $(P<0.05)$.

coincide con el mes más lluvioso que fue julio $(\mathrm{r}=0.80,0.86$ y 0.88 , respectivamente $)(P \leq 0.05)$; sin embargo, en todos los casos la correlación es fuerte, ya que a pesar que la asociación maíz-calabaza da mayor protección al suelo, los surcos de siembra están realizados a favor de la pendiente, por lo que el suelo es removido y trasladado con facilidad.

La pérdida de nutrientes depende de la cantidad de suelo erosionado y la concentración inicial de nutrientes (Morgan, 1997; Michelena, 2013); al inicio de la experimentación se realizó la caracterización físico-química de los suelos en los sistemas agrícolas evaluados (Cuadro 2), observando que hubo concentraciones de materia orgánica y nitrógeno bajas; el fósforo mostró cantidades medias en los cultivos de maíz-avena y calabaza; sin embargo, los valores fueron significativamente mayores en la parcela de maíz (18.8 $\left.\mathrm{mg} \mathrm{L}^{-1}\right)$ (NOM, 2000), debido a que el productor aplica periódicamente altas cantidades de abono orgánico (1500 Mg ha-1 , aproximadamente) (Pinos-Rodríguez, 2012).

Los sedimentos de los tres sistemas agrícolas

Cuadro 2. Propiedades físico-químicas de los suelos de los sistemas agrícolas evaluados.

\begin{tabular}{lccc}
\hline \multirow{2}{*}{ Propiedades } & \multicolumn{3}{c}{ Sistemas agrícolas } \\
\cline { 2 - 4 } \% Arena & Maíz & Maíz-Calabaza & Maíz-Avena \\
\% Arcilla & $42 \pm 0.07$ & $58 \pm 0.09$ & $28 \pm 0.03$ \\
\% Limo & $32 \pm 0.04$ & $22 \pm 0.05$ & $48 \pm 0.03$ \\
Clase textural & $26 \pm 0.04$ & $20 \pm 0.07$ & $24 \pm 0.04$ \\
pH $\left(\mathrm{H}_{2} \mathrm{O}\right)$ & Franco-arcilloso & Franco-areno-arcilloso & Arcilloso \\
Conductividad eléctrica $\left(\mathrm{dS} \cdot \mathrm{m}^{-1}\right)$ & $7.7 \pm 0.05$ & $7.1 \pm 0.01$ & $7.4 \pm 0.05$ \\
Fósforo disponible $\left(\mathrm{mg} \mathrm{L} \mathrm{L}^{-1}\right)$ & $1.0 \pm 0.02$ & $0.3 \pm 0.04$ & $0.3 \pm 0.01$ \\
Materia orgánica $(\mathrm{g} 100 \mathrm{~g})$ & $18.8 \pm 0.26$ & $10.7 \pm 0.41$ & $8.3 \pm 0.21$ \\
Nitrógeno total $(\mathrm{g} 100 \mathrm{~g})$ & $2.6 \pm 0.30$ & $1.3 \pm 0.03$ & $1.0 \pm 0.05$ \\
\hline Los & $0.4 \pm 0.08$ & $0.2 \pm 0.01$ & $0.1 \pm 0.04$ \\
\hline
\end{tabular}

Los resultados son el promedio de tres repeticiones \pm la desviación estándar. 
fueron caracterizados al final de la fase experimental, se encontraron ligeramente enriquecidos en la fracción arcilla, tuvieron la misma clase textural que el suelo inicial con excepción de la parcela de maíz que pasó de clase franco-arcillosa a arcillosa, el efecto de remoción y arrastre de las partículas más finas acumuló 11.7 \% de arcillas en sedimentos (Cuadro 3).

Los coeficientes promedio de enriquecimiento de los sedimentos erosionados (Cuadro 3, Figura 1) fueron en su mayoría superiores a uno, por tanto la erosión no sólo es un proceso cuantitativo de pérdidas de suelo, sino también cualitativo, ya que los sedimentos poseen mayor calidad físico-química que la matriz del suelo (Nadeu et al., 2010).

La pérdida de materia orgánica fue mayor $(P \leq 0.05)$ en maíz (8.9\%), maíz-avena y maízcalabaza no presentaron diferencias $(P \leq 0.05)$. El coeficiente de enriquecimiento de fósforo disponible fue superior $(P \leq 0.05)$ en la parcela de maíz-calabaza (1.14), mientras que los sedimentos de las parcelas de maíz y maíz-avena presentaron coeficientes de enriquecimiento parecidos, $0.17 \mathrm{y}$ 0.19 ; enriquecimientos de fósforo fueron menores a la unidad para las parcelas con maíz y maíz-avena, debido a la concentración inicial en el suelo asociada a la aplicación de estiércol. Similar comportamiento se presentó en el caso de nitrógeno, el coeficiente de enriquecimiento fue mayor $(P \leq 0.05)$ en la parcela de maíz-calabaza (2.70) que en maíz (1.18) y maíz-avena (1.20). En consecuencia, los resultados muestran que si bien la asociación maíz-calabaza impide el arrastre de partículas de suelo y materia orgánica, no sucede lo mismo con la pérdida de macronutrientes como fósforo y nitrógeno, debido a que las hojas anchas de la calabaza amortiguan el impacto de las gotas de lluvias, por lo que la disgregación y la desestabilización de la estructura del suelo es baja, sin embargo, no impide la disolución de nitrógeno y fósforo en el agua de escorrentía.

En el Cuadro 4 pueden observarse elevadas correlaciones entre producción de sedimentos y pérdidas de materia orgánica, nitrógeno y fósforo. Los tres sistemas evaluados presentaron alta correlación entre producción de sedimentos y pérdida de materia orgánica (0.97), la pérdida de nitrógeno presentó valores más altos con los cultivos de maíz-calabaza (0.98) y maíz-avena (0.98); y el fósforo disponible con cultivo de maíz (0.97).

La relación de la pérdida de materia orgánica, fósforo y nitrógeno, en función de la producción de sedimentos en los tres sistemas fue lineal, se muestra en las Figuras 2 y 3; en consecuencia al incrementarse la producción de sedimentos por efecto de la precipitación, se elevó el arrastre de los nutrientes del suelo. Las ecuaciones que relacionan las pérdidas de nutrientes y la producción de sedimentos en los sistemas agrícolas maíz-avena y maíz-calabaza presentaron la más baja $\mathrm{r}^{2}$ en los casos de pérdida de materia orgánica y fósforo; sin embargo, en nitrógeno la $\mathrm{r}^{2}$ fue mayor en maízcalabaza y maíz-avena, debido a que estos sistemas retienen por más tiempo el agua de escorrentía y se favorece la disolución de los nutrientes.

Cuadro 3. Propiedades físico-químicas de los sedimentos de los sistemas agrícolas evaluados.

\begin{tabular}{lccc}
\hline & & Sistemas agrícolas & \\
Propiedades & Maíz & Maíz-Calabaza & Maíz-Avena \\
\cline { 2 - 4 } \% Arena & $18 \pm 0.10$ & $58 \pm 0.05$ & $14 \pm 0.07$ \\
\% Arcilla & $44 \pm 0.11$ & $26 \pm 0.11$ & $60 \pm 0.05$ \\
\% Limo & $38 \pm 0.05$ & $16 \pm 0.05$ & $26 \pm 0.10$ \\
Clase textural & Arcilloso & Franco-areno-arcilloso & Arcilloso \\
Fósforo disponible (mg L-1) & $30.1 \pm 0.15$ & $12.2 \pm 0.05$ & $1.6 \pm 0.07$ \\
Coeficiente de enriquecimiento & $0.17 \mathrm{a}$ & $1.14 \mathrm{~b}$ & $0.19 \mathrm{a}$ \\
Materia orgánica (g 100g) & $8.9 \pm 0.10$ & $3.3 \pm 0.15$ & $2.6 \pm 0.17$ \\
Coeficiente de enriquecimiento & $3.42 \mathrm{a}$ & $2.6 \mathrm{~b}$ & $2.54 \mathrm{~b}$ \\
Nitrógeno total (g 100g) & $0.47 \pm 0.10$ & $0.24 \pm 0.03$ & $0.27 \pm 0.12$ \\
Coeficiente de enriquecimiento & $1.18 \mathrm{a}$ & $2.70 \mathrm{~b}$ & $1.20 \mathrm{a}$
\end{tabular}

Los resultados son el promedio de tres repeticiones \pm la desviación estándar. Letras distintas indican diferencias significativas $(P<0.05)$. 


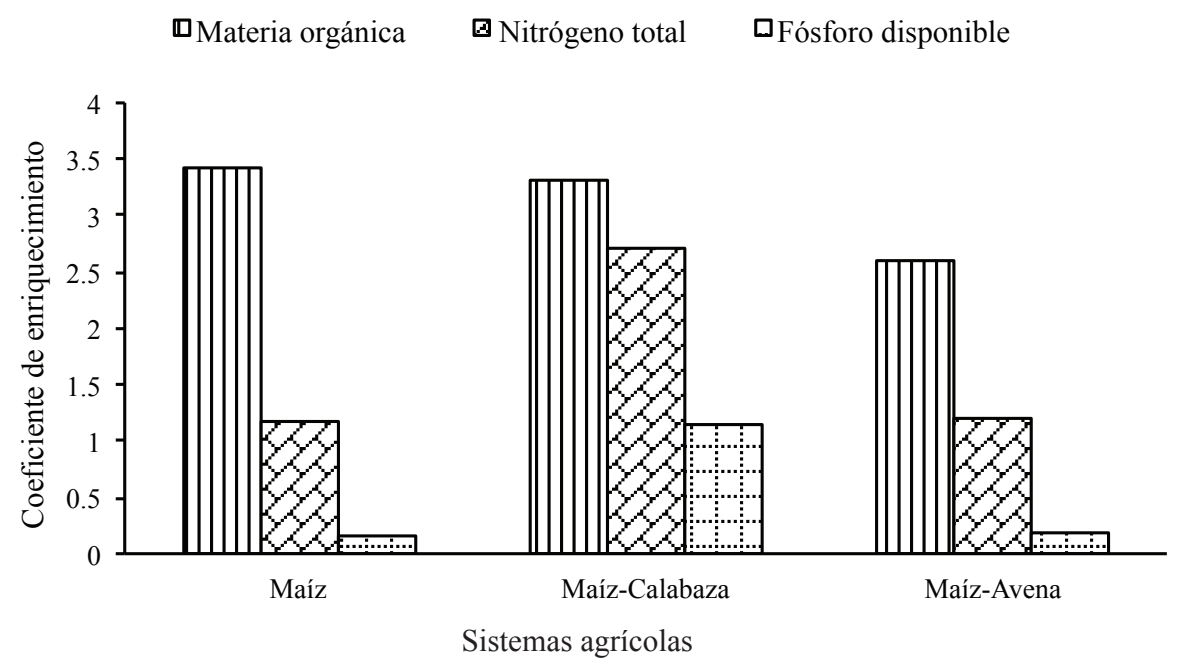

Figura 1. Coeficientes de enriquecimiento según el tipo de sistema agrícola.

Cuadro 4. Ecuaciones de regresión entre la producción de sedimentos, pérdida de materia orgánica, nitrógeno total y fósforo disponible.

\begin{tabular}{|c|c|c|c|c|}
\hline Sistema agrícola & Propiedad & $\mathrm{r}$ & $r^{2}$ & Ecuaciones de regresión \\
\hline & & & $\%$ & \\
\hline \multirow[t]{3}{*}{ Maíz } & Materia orgánica & 0.97 & 94.10 & $\begin{array}{l}\text { Pérdida de materia orgánica }(\mathrm{g} 100 \mathrm{~g})=0.667 * \text { producción de } \\
\text { sedimentos }\left(\mathrm{Mg} \mathrm{ha}^{-1}\right)+0.189\end{array}$ \\
\hline & Nitrógeno total & 0.97 & 94.60 & $\begin{array}{l}\text { Pérdida de nitrógeno total }(\mathrm{g} 100 \mathrm{~g})=0.23 * \text { producción de } \\
\text { sedimentos }\left(\mathrm{Mg} \mathrm{ha}^{-1}\right)+0.147\end{array}$ \\
\hline & Fósforo disponible & 0.97 & 95.90 & $\begin{array}{l}\text { Pérdida de fósforo disponible }\left(\mathrm{mg} \mathrm{L}^{-1}\right)=0.82 * \text { producción de } \\
\text { sedimentos }\left(\mathrm{Mg} \mathrm{ha}^{-1}\right)+19.48\end{array}$ \\
\hline \multirow[t]{3}{*}{ Maíz-Calabaza } & Materia orgánica & 0.96 & 92.30 & $\begin{array}{l}\text { Pérdida de materia orgánica }(\mathrm{g} 100 \mathrm{~g})=0.29 * \text { producción de } \\
\text { sedimentos }\left(\mathrm{Mg} \mathrm{ha}^{-1}\right)-0.494\end{array}$ \\
\hline & Nitrógeno total & 0.98 & 96.00 & $\begin{array}{l}\text { Pérdida de nitrógeno total }(\mathrm{g} 100 \mathrm{~g})=0.02 * \text { producción de } \\
\text { sedimentos }\left(\mathrm{Mg} \mathrm{ha}^{-1}\right)-0.009\end{array}$ \\
\hline & Fósforo disponible & 0.95 & 91.50 & $\begin{array}{l}\text { Pérdida de fósforo disponible }\left(\mathrm{mg} \mathrm{L}^{-1}\right)=0.48 * \text { producción de } \\
\text { sedimentos }\left(\mathrm{Mg} \mathrm{ha}^{-1}\right)+5.74\end{array}$ \\
\hline \multirow[t]{3}{*}{ Maíz-Avena } & Materia orgánica & 0.95 & 90.70 & $\begin{array}{l}\text { Pérdida de materia orgánica }(\mathrm{g} 100 \mathrm{~g})=0.52 * \text { producción de } \\
\text { sedimentos }\left(\mathrm{Mg} \mathrm{ha}^{-1}\right)-6.078\end{array}$ \\
\hline & Nitrógeno total & 0.98 & 95.90 & $\begin{array}{l}\text { Pérdida de nitrógeno total }(\mathrm{g} 100 \mathrm{~g})=0.06 * \text { producción de } \\
\text { sedimentos }\left(\mathrm{Mg} \mathrm{ha}^{-1}\right)-0.83\end{array}$ \\
\hline & Fósforo disponible & 0.95 & 90.30 & $\begin{array}{l}\text { Pérdida de fósforo disponible }\left(\mathrm{mg} \mathrm{L}^{-1}\right)=0.09 * \text { producción de } \\
\text { sedimentos }\left(\mathrm{Mg} \mathrm{ha}^{-1}\right)-0.204\end{array}$ \\
\hline
\end{tabular}



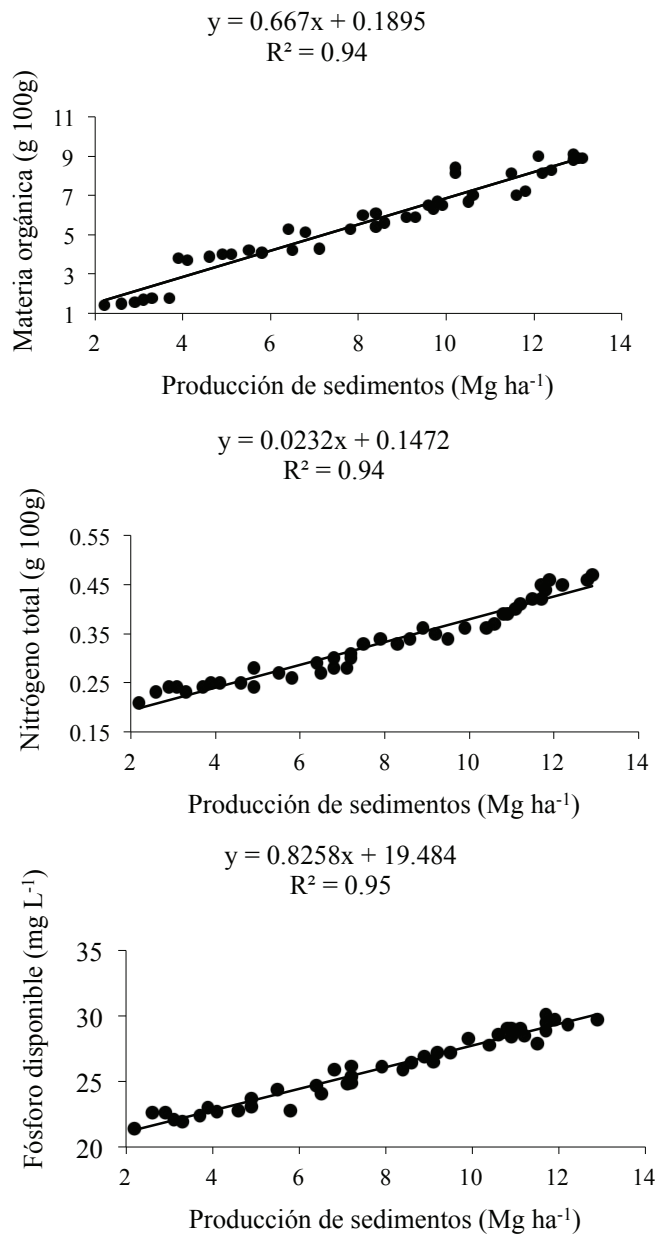
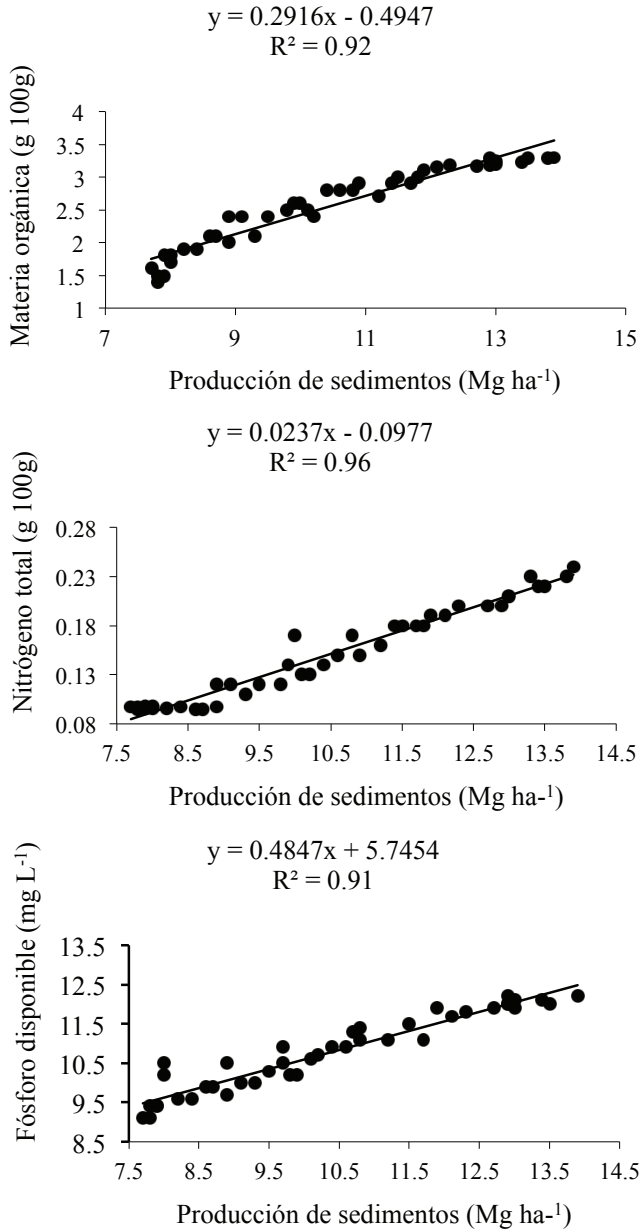

Figura 2. Regresión lineal entre la producción de sedimentos y las pérdidas de nutrientes para los sistemas agrícolas maíz y maíz-calabaza.
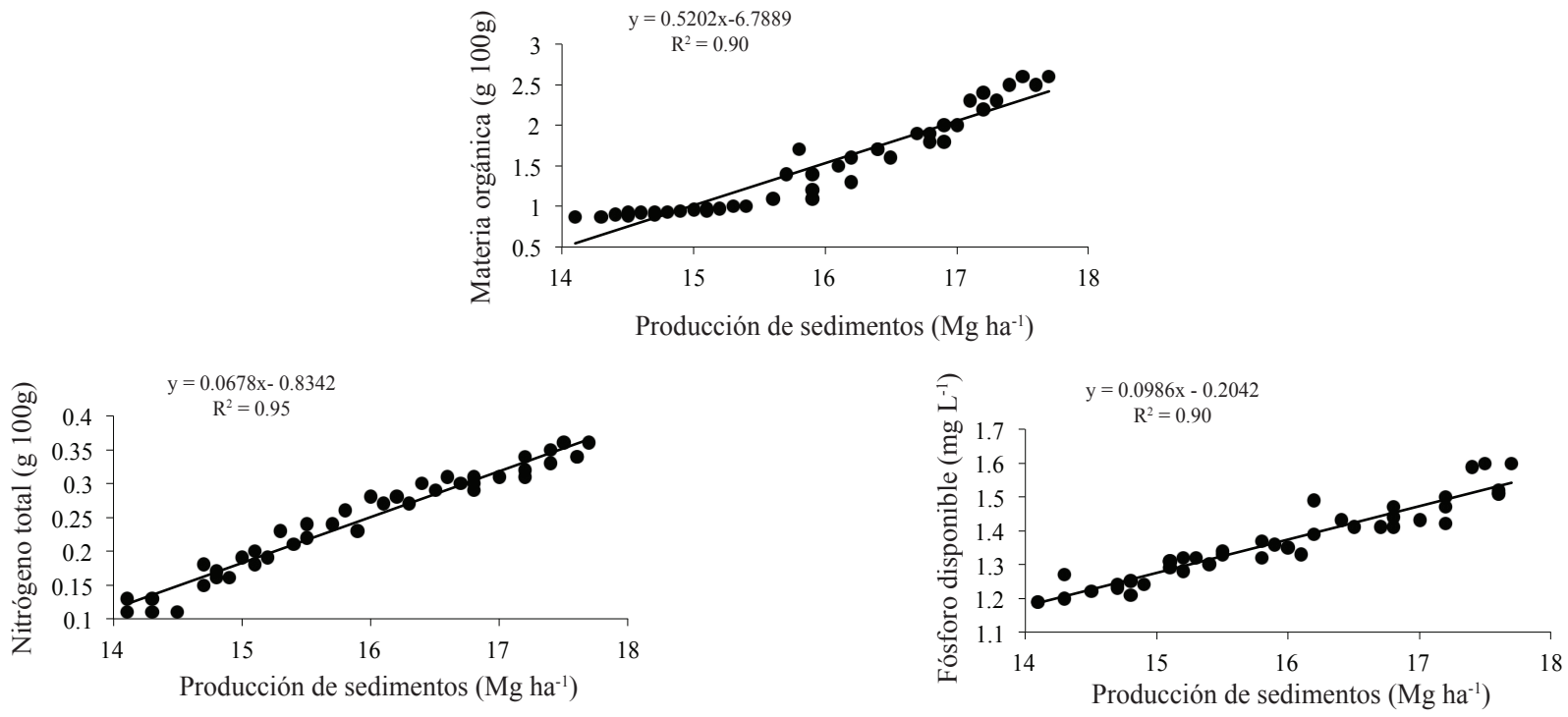

Figura 3. Regresión lineal entre la producción de sedimentos y las pérdidas de nutrientes para el sistema agrícola maíz-avena. 


\section{CONCLUSIONES}

- Se concluye que la producción de sedimentos y el volumen de escorrentía se correlacionan de forma importante con la precipitación. El cultivo de maíz sin protección es el que promueve en mayor medida la erosión del suelo, mientras que el sistema agrícola maíz-calabaza fue más eficiente para disminuir el arrastre de las partículas del suelo, pero no así para evitar la disolución de nutrientes como nitrógeno y fósforo en el agua de escorrentía. Los coeficientes de enriquecimiento de los sedimentos erosionados fueron, en su mayoría, superiores a la unidad, la materia orgánica tuvo los más altos coeficientes en los sistemas agrícolas estudiados.

- Los resultados obtenidos son importantes para establecer estrategias agrícolas para disminuir la degradación en zonas rurales de la ciudad de Puebla así como una evidencia de que los cultivos bajo las actuales formas de manejo, generan efectos negativos sobre el suelo por lo que se requiere promover manejos agrícolas sostenible.

\section{LITERATURA CITADA}

Adema, E. O., F. J. Babinec y N. Peinemann. 2001. Pérdida de nutrientes por erosión hídrica en dos suelos del Caldenal Pampeano. Cienc. Suelo 19: 144-154.

Almorox A., J., F. López B. y S. Rafaelli. 2010. La degradación de los suelos por erosión hídrica: Métodos de estimación. Universidad de Murcia. Editum. España.

Almoza, Y., H. Medina, W. Schiettecatte, G. Alonso y M. Ruiz. 2008. El uso de datos pluviométricos para la determinación del factor erosividad de las precipitaciones en el modelo RUSLE. Rev. Cienc. Téc. Agropec. 17: 38-43.

Bautista Z., F., H. Delfín G., J. L. Palacio P. y M. C. Delgado C. 2004. Técnicas de muestreo para manejadores de recursos naturales. UNAM, INE, CONACYT, UAY. México.
Cotler, H., C. A. López y S. Martínez-Trinidad. 2001. ¿Cuánto nos cuesta la erosión de suelos? Aproximación a una valoración económica de la pérdida de suelos agrícolas en México. Invest. Amb. Cienc. Pol. Púb. 3: 31-43.

Den Biggelaar, C., R. Lal, K. Wiebe, and V. Breneman. 2004. The global impact of soil erosion on productivity. 1: Absolute and relative erosion-induced yield losses. Adv. Agron. 81: 1-48.

FAO (Organización de las Naciones Unidas para la Alimentación y la Agricultura). 1997. Lucha contra la contaminación agrícola de los recursos hídricos. Departamento de Desarrollo Sostenible (Estudio FAO Riego y Drenaje - 55). Roma, Italia.

INEGI (Instituto Nacional de Estadística y Geografía). 2000. Síntesis geográfica del estado de Puebla y anexo cartográfico. México. ISBN 970-13-2365-3.

Leal, Z., J. Díaz, W. Schiettecatte, M. E. Ruiz y Y. Almoza. 2007. Efecto de la cobertura vegetal de cultivos agrícolas principales sobre el proceso de erosión en suelos de la cuenca del río Cuyaguateje. Cienc. Téc. Agropec. 16: 76-83.

Lobo, G. P. and C. A. Bonilla. 2015. Sensitivity analysis of kinetic energy-intensity relationships and maximum rainfall intensities on rainfall erosivity using a long-term precipitation dataset. J. Hydrol. 527: 788-793.

Michelena, R. 2013. Cómo controlar la erosión hídrica para que no destruya los suelos. Instituto de Suelos INTA Castelar. Buenos Aires, Argentina.

Morgan, R. 1997. Erosión y conservación del suelo. Mundi Prensa. Madrid, España.

Nadeu, E., C. Boix Fayos, J. de Vente, J. López y M. Martínez. 2010. Movilización de carbono orgánico por distintos procesos erosivos en la conexión ladera-cauce. Pirineos-Rev. Ecol. Montaña 165: 157-177.

NOM-021-SEMARNAT-2000. 2000. Norma Oficial Mexicana que establece las especificaciones de fertilidad, salinidad y clasificación de suelos, estudio, muestreo y análisis. Secretaria de medio ambiente y recursos naturales. México, D. F.

Pinos-Rodríguez, J. M., J. C. García-López, L. Y. Peña-Avelino, J. A. Rendón-Huerta, C. González-González y F. Tristán-Patiño. 2012. Impactos y regulaciones ambientales del estiércol generado por los sistemas ganaderos de algunos países de América. Agrociencia 46: 359-370.

Rivera, J. H. y A. A. Gómez. 1991. Erosividad de las lluvias de la zona cafetalera central colombiana (Caldas, Quindío, Risaralda). Cenicafé 42: 37-52.

Sharpley, A. N. 1985. The selective erosion of plant nutrients in runoff. Soil Sci. Soc. Am. J. 49: 1527-1534. 